\title{
The effect of contact length on adhesion between carbon nanotubes on silicon dioxide
}

\author{
Robert C. Davis \\ Kaylee McElroy \\ Aaron R. Hopkins \\ hawkins@ee.byu.edu
}

Follow this and additional works at: https://scholarsarchive.byu.edu/facpub

Part of the Electrical and Computer Engineering Commons

\section{Original Publication Citation}

Mcelroy, Kaylee, Robert C. Davis, and Aaron Hawkins. "The effect of contact length on adhesion between carbon nanotubes on silicon dioxide." Applied Physics Letters 91 (27)

\section{BYU ScholarsArchive Citation}

Davis, Robert C.; McElroy, Kaylee; and Hopkins, Aaron R., "The effect of contact length on adhesion between carbon nanotubes on silicon dioxide" (2007). Faculty Publications. 218.

https://scholarsarchive.byu.edu/facpub/218 


\title{
The effect of contact length on adhesion between carbon nanotubes on silicon dioxide
}

\author{
Kaylee McElroy and Robert C. Davis \\ Department of Physics and Astronomy, Brigham Young University, Provo, Utah 84602, USA \\ Aaron Hawkins ${ }^{\text {a) }}$ \\ Department of Electrical and Computer Engineering, Brigham Young University, Provo, Utah 84602, USA
}

(Received 9 June 2007; accepted 13 November 2007; published online 7 December 2007)

\begin{abstract}
The force of adhesion was measured for single walled carbon nanotubes grown over lithographically defined silicon dioxide trenches. We varied contact lengths between the nanotubes and silicon dioxide from 230 to $850 \mathrm{~nm}$. Suspended nanotubes were pushed vertically into the trenches with an atomic force microscope tip, causing them to slip along the surface. Previous work done at shorter contact lengths found that tension was constant with contact length [J. D. Whittaker et al., Nano Lett. 6, 953 (2006)]. This study finds that when the nanotube contact length approaches $1 \mu \mathrm{m}$, the tension at which nanotubes slip begins to increase with contact length. This indicates that contact length independent adhesion is a uniquely nanoscale behavior. (C) 2007 American Institute of Physics. [DOI: 10.1063/1.2821228]
\end{abstract}

Adhesion plays a significant role in many nanotube based composites and in single nanotube based nanomechanical devices. ${ }^{1,2}$ However, there are few controlled direct measurements of nanotube adhesion. In particular, the contact length dependence of the adhesion has been studied only over a small range. Previously, Akita et al. estimated the force of adhesion of carbon nanotubes (CNTs) by pulling a CNT off the $120 \mathrm{~nm}$ side of a digital versatile disk pit. ${ }^{3}$ Whittaker et al. measured the force of adhesion by applying a vertical force on a suspended section of CNT with an atomic force microscope (AFM) probe, causing the tube to slip along its axis, as illustrated in Fig. 1. They found that the tension at which the CNTs slipped was constant over the range of contact lengths from 140 to $246 \mathrm{~nm}$. We expanded on this work by measuring the force of adhesion between CNTs and $\mathrm{SiO}_{2}$ for a greater range of contact lengths. We extended this range upward to $850 \mathrm{~nm}$ and found the adhesion force to increase with length from 230 to $850 \mathrm{~nm}$.

To precisely measure the nanotube contact length, nanotubes were grown across and measured on trench structures, as described previously. ${ }^{4}$ Briefly, trenches were fabricated on silicon wafers using standard electron-beam lithography and dry etching techniques. The trenches were 100-250 nm wide and $80-125 \mathrm{~nm}$ deep. Nanotubes were grown across the trenches using chemical vapor deposition. The nanotubes were $0.7-2.7 \mathrm{~nm}$ in diameter, as measured by AFM.

Atomic force microscopy was used to image and perform force-distance measurements on the suspended tubes (Fig. 1). The AFM measurements were performed in air with a Veeco Nanoscope Dimension 3100 AFM. The scan size was limited so that one suspended CNT was positioned at the center of the $X Y$ scan area. After scanning over the CNT for 5-10 min to ensure that drift was minimized, several forcedistance curves were taken, each applying an increasing amount of force on the CNT until slipping occurred. Determination of the slipping location was made using AFM imaging to identify movement of the nanotube slack from one

\footnotetext{
${ }^{a)}$ Electronic mail: davis@byu.edu.
}

suspended region to another as a result of a push (see Fig. 2). The contact length where the slipping occurred was determined by measuring the length of nanotube contact with $\mathrm{SiO}_{2}$ between the trench that originally had slack and the trench that had slack after pushing. The segments of nanotubes that were pushed were chosen such that only one neighboring trench had slack. Although some small slipping could have occurred in the other direction, we only included the large slipping events that resulted in movement of the slack in our data.

The force $F$ applied with the AFM probe and the tension $T$ of the nanotube were calculated as follows:

$$
F=k_{\text {tip }} z_{\text {defl }}
$$

and

$$
T=\frac{F}{2 \sin \theta},
$$

where $k_{\text {tip }}$ is the spring constant of the cantilever and $z_{\text {defl }}$ is the distance the tip is deflected at the slip event. The spring constant for each cantilever was determined using the geometry and frequency of the cantilever as well as its density and

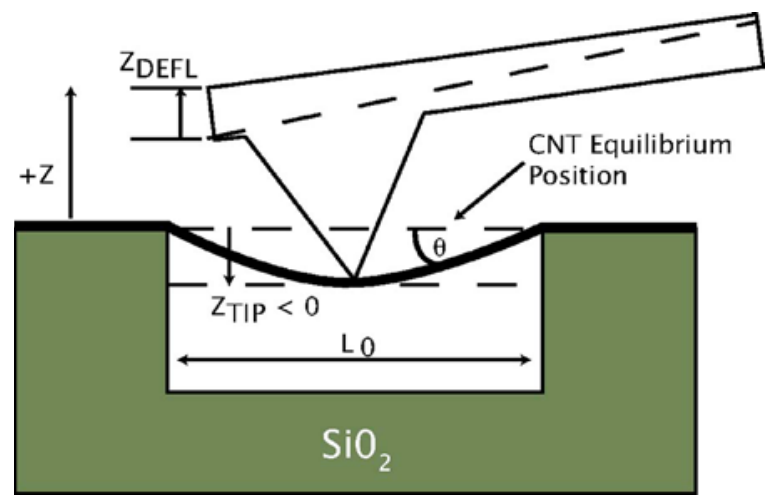

FIG. 1. (Color online) The AFM probe applies a force at the center of the suspended portion of the CNT. When the force is great enough, the CNT will slip along the $\mathrm{SiO}_{2}$ surface. $z_{\text {tip }}=z_{\text {piezo }}-z_{\text {deflection }}$ due to the bending of the cantilever. 


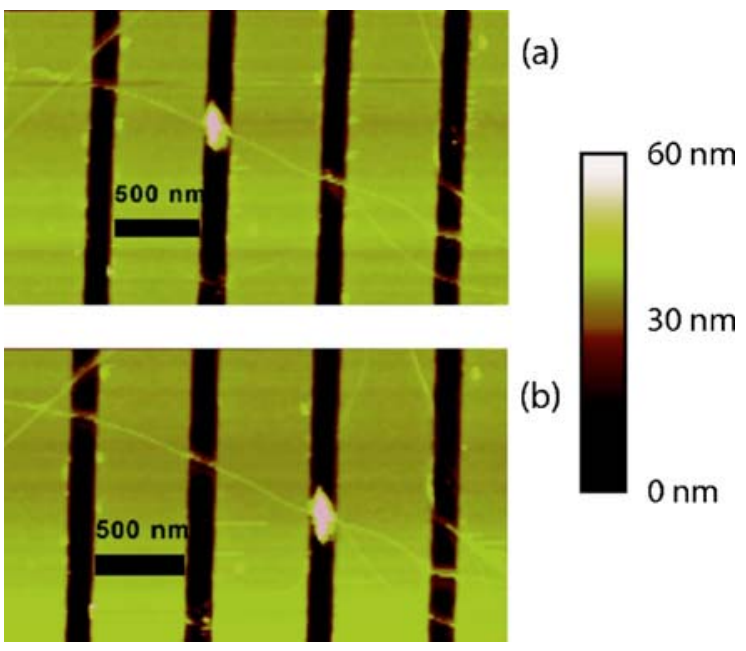

FIG. 2. (Color online) Slack was transferred from one suspended CNT segment to another. (a) The CNT before the push. Slack is over the centerleft trench. (b) The CNT after the center-right CNT segment was pushed.

elastic modulus. ${ }^{5,6}$ For the calculation of $k_{\text {tip }}$ for Veeco NP cantilevers that are $115 \mu \mathrm{m}$ long and $17 \mu \mathrm{m}$ wide, we used $E=280 \mathrm{GPa}$ and $\rho_{\text {silicon nitride }}=3100 \mathrm{~kg} / \mathrm{m}^{3}$ and found the spring constant to be $0.12 \mathrm{~N} / \mathrm{m}$. For MikroMasch CSC11 A cantilevers, we used $E=131 \mathrm{GPa}$ and $\rho_{\text {silicon }}=2330 \mathrm{~kg} / \mathrm{m}^{3}$ and found the spring constant to be $0.19 \mathrm{~N} / \mathrm{m}$. These are the same densities and elastic moduli used in Ref. $4 .^{7}$
This method of measuring the force of adhesion assumes that all the force applied to the CNT is used to make the CNT slip and that no force is required to make the CNT bend. This approximation is based on the following observation: van der Waals forces between the AFM tip and a CNT with slack are sufficient to cause the CNT to jump up and contact the tip while the AFM is imaging. This is seen in AFM images where a suspended segment of the CNT appears to be higher than the top of the trenches (see Fig. 2). The force applied by the tip while imaging is much smaller than the force required for slipping, which indicates that the bending force is insignificant compared to the force of CNT adhesion.

Analysis of the amplitude and deflection curves obtained in force-distance mode can be used to distinguish between three types of pushing events: ${ }^{4}$ (I) the CNT slipped along the surface, (II) the AFM tip stayed on the CNT but did not push hard enough for the CNT to slip along the $\mathrm{SiO}_{2}$ surface, and (III) the AFM tip slipped along the surface of the CNT. We will not consider data of type III.

When the AFM tip stayed on the CNT but the CNT did not slip (type II), the extension and retraction traces are aligned in the amplitude versus distance and force versus distance graphs. The amplitude traces have peaks in them due to the slack in the CNT [see Fig. 3(a)]. As the tip is extended, the CNT jumps up into contact with the tip, causing the amplitude to suddenly drop off. As the extension of the tip continues, the slack in the CNT allows the tip to

\section{(a) no slip event}
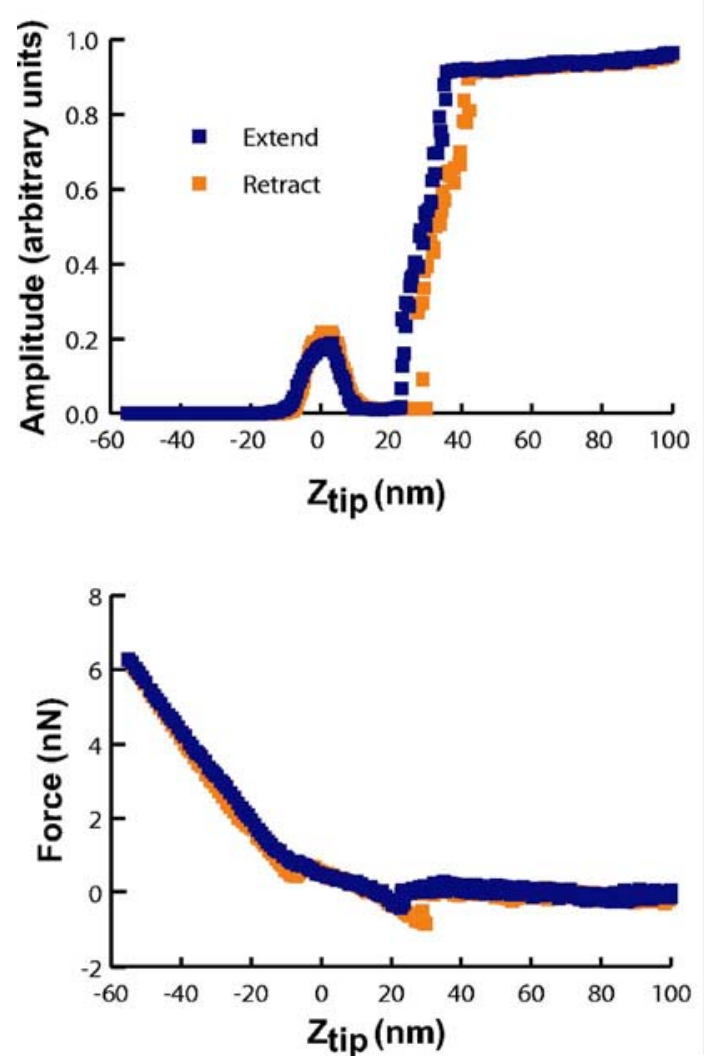

(b) CNT slip

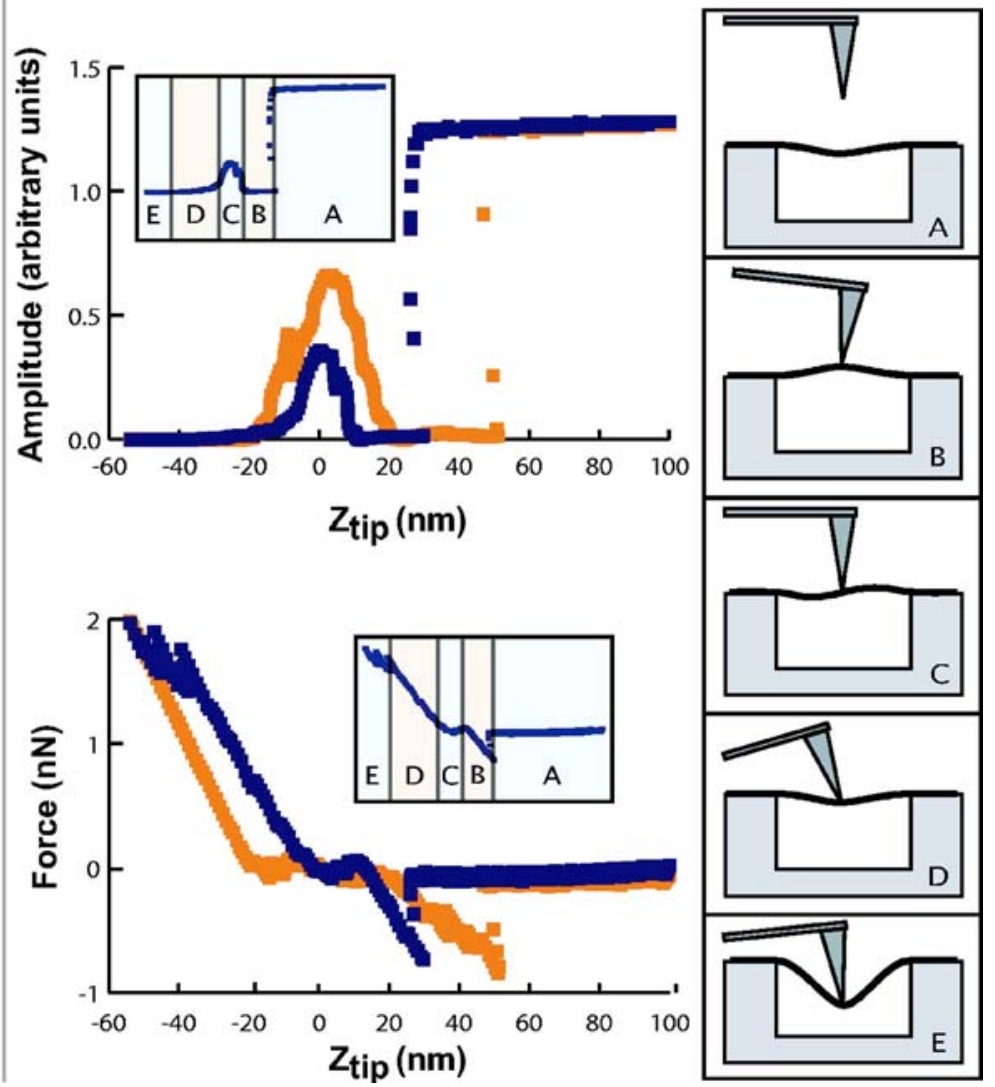

FIG. 3. (Color online) (a) Amplitude and force graphs when the CNT does not slip. The extension and retraction traces follow each other closely. (b) Amplitude and force graphs of a CNT slip. The taller peak in the retraction trace is caused by the slack added to the CNT. The slip event is seen at $-35 \mathrm{~nm}$ on the $z_{\text {tip }}$ axis on the extension trace of the force graph. Insets separate the graphs into sections depending on tip-tube interaction, and each section is represented graphically in (c).

Downloaded 10 Feb 2009 to 128.187.0.164. Redistribution subject to AIP license or copyright; see http://apl.aip.org/apl/copyright.jsp 


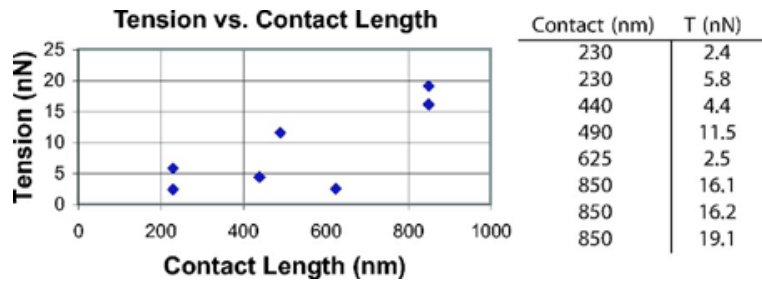

FIG. 4. (Color online) Tension plotted against the CNT's contact length with the $\mathrm{SiO}_{2}$. The data are also listed in a table, showing that three measurements were made at the $850 \mathrm{~nm}$ contact length.

momentarily oscillate before the amplitude drops off again as more force is applied to the CNT, making the CNT taut. The location of the peak center along the $Z_{\text {tip }}$ axis corresponds to the $z$-axis position of the top of the trenches; when the tip is at this position, there is maximum slack in the tube.

Force-distance curves for a slip event (type I) also have peaks in both traces of the amplitude curves but in this case, the peak in the retraction trace is higher than that in the extension trace because there is more slack in the CNT during the retraction. The slip event is seen in the force curve. As the AFM starts to put force on the CNT, the force signal rises. When the CNT slips, a sudden decrease or leveling off occurs in the force signal because of the extra slack suddenly added to the suspended section of the CNT [see Fig. 3(b)]. The slip in Fig. 3(b) occurred at $-35 \mathrm{~nm}$ along the $z_{\text {tip }}$ axis. The extra slack added to a CNT could often be seen in the AFM imaging by comparing pictures taken before and after a push (see Fig. 2).

The resulting tensions ranged from 2.4 to $19.1 \mathrm{nN}$. A graph of the tension versus contact length (see Fig. 4) indicates that the tension required for the CNT to slip depends on the contact length of the CNT.

Despite the scatter in the data, the statistical significance of the correlation here is quite strong. Using each contact length value $x_{i}$ and corresponding tension value $y_{i}$, the correlation coefficient $r$ was calculated as follows:

$$
r=\frac{\sum\left(x_{i}-\bar{x}\right)\left(y_{i}-\bar{y}\right)}{\left[\sum\left(x_{i}-\bar{x}\right)^{2} \sum\left(y_{i}-\bar{y}\right)^{2}\right]^{1 / 2}},
$$

where $\bar{x}$ is the average contact length and $\bar{y}$ is the average tension. ${ }^{8}$ The eight data points found in this experiment show that contact length and tension have a correlation coefficient of 0.81 . The probability of having a correlation coefficient greater than or equal to 0.8 from eight random data points is $1.7 \%$.

The force of adhesion in macroscopic systems generally follows:

$$
F=\mu L+c A
$$

where $\mu$ is the frictional coefficient, $L$ is the load, $c$ is a constant coefficient, and $A$ is the contact area. ${ }^{9}$ The increase in adhesion we observe as the contact length increases toward $1 \mu \mathrm{m}$ can be explained by this macroscopic model. However, at lengths shorter than $0.5 \mu \mathrm{m}$ we do not see a significant length dependence in contrast with this macroscopic model but in agreement with the previous work. Whittaker et al. observed that the tension at which CNT slip is constant over a range of contact length from 140 to $246 \mathrm{~nm}$. Our experiment shows that tension increases with contact length when contact length is varied from 230 to $850 \mathrm{~nm}$. This change from nanoscale to microscale behavior indicates a transition of the adhesion mechanism. Niederberger et al. also observed adhesion behavior changes when comparing microscale and nanoscale contact areas. ${ }^{10}$ However, in their experiments, contact areas could only be very roughly estimated precluding a quantitative determination of frictional force versus area.

In summary, our measurements show that nanotube adhesion increases by a factor of 4 as the contact length increases from 230 to $850 \mathrm{~nm}$. This is in contrast with measurements on the 100-200 $\mathrm{nm}$ range where the adhesion force is independent of contact length. This change from weak to strong dependence on contact length indicates a change in the adhesion mechanism as the contact length changes from the nanoscale to the microscale.

This work was supported by the National Science Foundation Center for Nanoscale Systems at Cornell University and by Brigham Young University Undergraduate Mentoring.

${ }^{1}$ M. Falvo, G. Clary, A. Helse, S. Paulson, R. M. Taylor, V. Chi, F. P. Brooks, S. Wasburn, and R. Superfine, Microsc. Microanal. 4, 504 (1999).

${ }^{2}$ T. Hertel, R. Martel, and P. Avouris, J. Phys. Chem. B 102, 910 (1998).

${ }^{3}$ S. Akita, H. Nishijima, T. Kishida, and Y. Nakayama, Jpn. J. Appl. Phys., Part 1 39, 3724 (2000).

${ }^{4}$ J. D. Whittaker, E. D. Minot, D. Tanenbaum, P. L. McEuen, and R. C. Davis, Nano Lett. 6, 953 (2006).

${ }^{5}$ J. P. Cleveland, S. Manne, D. Bocek, and P. K. Hansma, Rev. Sci. Instrum. 64, 403 (1993).

${ }^{6}$ H.-J. Butt, P. Siedle, K. Seifert, K. Fendler, T. Seeger, E. Bamberg, A. L. Weisenhorn, K. Goldie, and A. Engel, J. Microsc. 169, 75 (1993).

${ }^{7}$ J. D. Whittaker (private communication).

${ }^{8}$ J. R. Taylor, An Introduction to Error Analysis: The Study of Uncertainties in Physical Measurements (University Science Books, Mill Valley, Califonia, 1982), pp. 173-185, 248-249.

${ }^{9}$ J. Ringlein and M. O. Robbins, Am. J. Phys. 72, 884 (2004).

${ }^{10}$ S. Niederberger, D. H. Gracias, K. Komvopoulos, and G. A. Somorjai, J. Appl. Phys. 87, 3143 (2000). 